\title{
Significant association between parathyroid hormone and uric acid level in men
}

This article was published in the following Dove Press journal:

Clinical Interventions in Aging

21 August 2015

Number of times this article has been viewed

\author{
Kok-Yong Chin' \\ Soelaiman Ima Nirwana' \\ Wan Zurinah Wan Ngah ${ }^{2}$ \\ 'Department of Pharmacology, \\ ${ }^{2}$ Department of Biochemistry, Faculty \\ of Medicine, Universiti Kebangsaan \\ Malaysia Medical Centre, Kuala \\ Lumpur, Malaysia
}

Background: Previous reports of patients undergoing parathyroidectomy and of patients receiving teriparatide as antiosteoporotic treatment have suggested a plausible relationship between parathyroid hormone (PTH) and uric acid. However, similar data at population level were lacking. The current study aimed to determine the relationship between PTH and uric acid in a group of apparently healthy Malaysian men.

Methods: A cross-sectional study was conducted among 380 Malay and Chinese men aged 20 years and above, residing in the Klang Valley, Malaysia. Their body anthropometry was measured, and their fasting blood samples were collected for biochemical analysis. The relationship between PTH and uric acid was analyzed using regression analysis.

Results: Increased serum PTH level was significantly associated with increased serum uric acid level $(\beta=0.165 ; P=0.001)$. Increased PTH level was also significantly associated with the condition of hyperuricemia in the study population (odds ratio [OR], $1.045 ; 95 \%$ confidence interval $[\mathrm{CI}], 1.017-1.075 ; P=0.002$ ). All analyses were adjusted for age, body mass index, vitamin $\mathrm{D}$, total calcium, inorganic phosphate, blood urea nitrogen and creatinine levels.

Conclusion: There is a significant positive relationship between PTH level and uric acid level in Malaysian men. This relationship and its clinical significance should be further investigated in a larger longitudinal study.

Keywords: hyperuricemia, Asian, cross-sectional study, uric acid, urate

\section{Introduction}

Hyperuricemia is a predisposing factor for gout, which is a common inflammatory joint disease affecting $0.08 \%$ of the population worldwide. ${ }^{1}$ Previous clinical studies have uncovered a significant association between parathyroid hormone (PTH) and uric acid level in humans. ${ }^{2,3}$ Hyperuricemia has been found in patients suffering from hyperparathyroidism. ${ }^{3}$ Uric acid level has been found to decrease after parathyroidectomy. ${ }^{4}$ Teriparatide, a recombinant PTH used as antiosteoporotic agent, has been found to increase the incidence of hyperuricemia in a dose-dependent manner. ${ }^{5}$ However, there is a paucity of data on the association between PTH and uric acid in the general population. Only two reports from the US National Health and Nutritional Examination Survey (NHANES) have confirmed the significant relationship between PTH and uric acid. ${ }^{6,7}$ To the best of our knowledge, no Asian data have been reported so far. Hence, the aim of the present study is to determine the relationship between PTH and uric acid in the Malaysian population.

\section{Materials and methods}

This cross-sectional study was conducted from September 2009 to September 2011. It adopted a purposive sampling technique, whereby subjects were solicited via announcement with specific inclusion and exclusion criteria advertised via 
major newspapers, radio broadcast, religious venues, and community centers. Subjects recruited were Chinese and Malay men aged 20 years and above, residing in the Klang Valley, Malaysia. ${ }^{8}$ They were not previously diagnosed with major debilitating diseases or gout. None of them reported the use of allopurinol and/or uricosuric agents. The subjects gave written consent before enrolling in the study. The study protocol has been reviewed and approved by Research Ethics Committee of Universiti Kebangsaan Malaysia Medical Centre.

Age of the subjects was determined from the date of birth recorded on their identification cards. Ethnicity of the subjects was self-declared. Height of the subjects was measured using a Seca 213 stadiometer (Seca GmbH, Hamburg, Germany) and was recorded to the nearest $1 \mathrm{~cm}$. Body weight of the subjects was measured using BC-418 Segmental Body Composition Analyzer (Tanita Corporation, Tokyo, Japan) and was recorded to the nearest $0.1 \mathrm{~kg}$. Body mass index was calculated as per the convention. Blood of the subjects was collected between 8.30 am and 10.30 am after an overnight fast. Serum was extracted and sent for biochemical analysis. Serum uric acid, total calcium, inorganic phosphate, blood urea nitrogen (BUN) and creatinine levels were determined using automated chemistry analyzer (Advia 2400; Siemens Healthcare Diagnostics, Erlangen, Germany). Serum PTH (IBL International, Hamburg, Germany) and 25-hydroxyvitamin D (IDS plc, Tyne and Wear, UK) levels were determined using enzyme-linked immunoassay kits. The interassay coefficient of variance values were $1.15 \%-2.41 \%$ for uric acid, $1.10 \%-1.17 \%$ for total calcium, $0.86 \%-1.19 \%$ for inorganic phosphate, $0.96 \%-1.69 \%$ for BUN, $2.84 \%-3.47 \%$ for creatinine, $2.80 \%-3.60 \%$ for intact PTH, and $4.60 \%-8.70 \%$ for 25-hydroxyvitamin D.

\section{Statistical analysis}

Normality of the data was assessed using the KolmogorovSmirnov test. Comparison of the characteristics between normal and hyperuricemic subjects was performed using independent $t$-test if the data were normally distributed or the Mann-Whitney $U$-test if the data were skewed. The association between serum uric acid and PTH level was determined using multiple linear regression analysis. Multivariate outliers were identified using standardized residual and Mahalanobis distance. The subjects were subsequently divided into normal ( $\leq 0.42 \mathrm{mmol} / \mathrm{L})$ and hyperuricemic groups $(>0.42 \mathrm{mmol} / \mathrm{L})$. The association between the condition of hyperuricemia and PTH level was determined using binary logistic regression. Multivariate outliers were identified using standardized residual and Cook's distance. Confounding factors such as age, body mass index, ethnicity, and serum vitamin D level, BUN, and creatinine level were adjusted for both regression models. Statistical significance was set at $P<0.05$. Statistical analysis was performed using Statistical Package for the Social Science version 20.0 (IBM Corporation, Armonk, NY, USA).

\section{Results}

Data from 380 subjects (mean age, 43.0 [SD 15.5] years) were included in the final analysis. None of the subjects had abnormal total calcium level. Hyperuricemic subjects constituted $31.84 \%$ of the total recruitment. Body weight, body mass index, serum PTH, BUN, and creatinine level were significantly lower in the hyperuricemic subjects compared to the normal subjects $(P<0.05)$ (Table 1$)$. In the multiple linear regression model, the relationship between serum PTH and uric acid was positive, independent of BUN and creatinine level $(\beta=0.165 ; P=0.001 ; n=369$ after eliminating multivariate outliers) (Table 2). Similarly, hyperuricemia was positively and significantly associated with increased

Table I Characteristics of the subjects

\begin{tabular}{|c|c|c|c|c|c|c|c|}
\hline & \multicolumn{2}{|c|}{ Total $(n=380)$} & \multicolumn{2}{|c|}{ Normal $(n=334)$} & \multicolumn{2}{|c|}{ Hyperuricemic $(n=46)$} & \multirow[t]{2}{*}{$P$-value } \\
\hline & Mean & SD & Mean & SD & Mean & SD & \\
\hline Age (years) & 43.0 & 15.5 & 42.8 & 15.5 & 44.1 & 14.9 & 0.606 \\
\hline Height (cm) & 167.5 & 6.4 & 167.5 & 6.6 & 167.6 & 5.5 & 0.982 \\
\hline Weight (kg) & 68.4 & 12.9 & 67.2 & 12.4 & 77.1 & 13.5 & $<0.001$ \\
\hline Body mass index $\left(\mathrm{kg} / \mathrm{m}^{2}\right)$ & 24.3 & 4.2 & 23.9 & 4.0 & 27.4 & 4.2 & $<0.001$ \\
\hline Serum uric acid (mmol/L) & 0.4 & 0.1 & 0.4 & 0.1 & 0.5 & 0.1 & $<0.001$ \\
\hline Serum 25(OH)D (nmol/L) & 58.8 & 12.3 & 58.6 & 12.5 & 60.5 & II.I & 0.324 \\
\hline Serum total calcium (mmol/L) & 2.3 & 0.1 & 2.3 & 0.1 & 2.3 & 0.1 & 0.563 \\
\hline Serum inorganic phosphate $(\mathrm{mmol} / \mathrm{L})^{*}$ & I.I & 0.2 & I.I & 0.1 & I.I & 0.2 & 0.093 \\
\hline Serum creatinine $(\mu \mathrm{mol} / \mathrm{L})$ & 82.4 & 15.6 & 81.1 & 14.7 & 92.0 & 17.9 & 0.001 \\
\hline Serum blood urea nitrogen $(\mathrm{mmol} / \mathrm{L})$ & 4.3 & 1.0 & 4.2 & 1.0 & 4.8 & 1.3 & $>0.001$ \\
\hline Serum intact PTH $(\mathrm{ng} / \mathrm{mL})^{*}$ & 45.4 & 19.0 & 44.0 & 17.7 & 55.8 & 24.0 & $<0.001$ \\
\hline
\end{tabular}

Notes: *Skewed data analyzed using the Mann-Whitney U-test. The other data were analyzed using the independent $t$-test.

Abbreviations: SD, standard deviation; PTH, parathyroid hormone; 25(OH)D, 25-hydroxyvitamin D. 
Table 2 The association between serum uric acid and PTH level indicated by multiple linear regression

\begin{tabular}{|c|c|c|c|c|}
\hline Predictor & $\begin{array}{l}\text { Unstandardized } \\
\text { regression } \\
\text { coefficient }\end{array}$ & $\begin{array}{l}\text { Standard } \\
\text { error }\end{array}$ & $\begin{array}{l}\text { Standardized } \\
\text { regression } \\
\text { coefficient }\end{array}$ & $P$-value \\
\hline Parathyroid & 0.001 & $<0.001$ & 0.165 & 0.001 \\
\hline $\begin{array}{l}\text { Blood urea } \\
\text { nitrogen }\end{array}$ & 0.007 & 0.004 & 0.096 & 0.063 \\
\hline Creatinine & 0.001 & $<0.001$ & 0.239 & $<0.001$ \\
\hline
\end{tabular}

Notes: The association between serum uric acid and PTH level was investigated using multiple linear regression, with adjustment for age, body mass index, ethnicity, serum vitamin D, blood urea nitrogen, and creatinine level. Serum PTH was significantly associated with uric acid level independent of indicators of renal function in the subjects.

Abbreviation: PTH, parathyroid hormone.

serum PTH level, independent of BUN and creatinine level in the binary logistic regression model (OR, 1.045; CI, $1.017-1.075 ; P=0.002 ; \mathrm{n}=360$ after eliminating multivariate outliers) (Table 3 ). The results suggested that the relationship between serum uric acid and PTH was independent of the renal function of the subject. Both the linear and binary regression analyses were adjusted for age, body mass index, ethnicity, serum vitamin D, BUN, and creatinine level.

\section{Discussion}

The present study found that PTH was positively and significantly associated with uric acid level and hyperuricemia after adjusting for potential confounders such as age, body mass index, ethnicity, vitamin D, BUN, and creatinine level. This finding was in line with the NHANES reports by Hui et a ${ }^{6}$ ( 8,316 subjects aged 18 years and above) and the report by Paik et al ${ }^{7}$ (7,652 subjects aged above 12 years), in which subjects in the higher quartiles/quintiles had significantly higher uric acid level compared to subjects in the lowest quartile/ quintile after adjustment for potential confounders.

The evidence on the association between PTH and uric acid was derived primarily from hyperparathyroid cases. Bergenfelz et al ${ }^{4}$ (323 patients operated on for sporadic primary

Table 3 The association between hyperuricemia and PTH level indicated by binary logistic regression

\begin{tabular}{|c|c|c|c|c|}
\hline Predictor & $\begin{array}{l}\text { Odds } \\
\text { ratio }\end{array}$ & $\begin{array}{l}\text { Lower-bound } \\
\text { confidence } \\
\text { interval }\end{array}$ & $\begin{array}{l}\text { Upper-bound } \\
\text { confidence } \\
\text { interval }\end{array}$ & $P$-value \\
\hline Parathyroid & 1.045 & 1.017 & 1.075 & 0.002 \\
\hline $\begin{array}{l}\text { Blood urea } \\
\text { nitrogen }\end{array}$ & 2.892 & 1.506 & 5.556 & 0.001 \\
\hline Creatinine & 1.161 & 1.095 & 1.23 & $<0.001$ \\
\hline
\end{tabular}

Notes: The association between hyperuricemia and PTH level was investigated using binary logistic regression, with adjustment for age, body mass index, ethnicity, serum vitamin $D$, blood urea nitrogen, and creatinine level. Hyperuricemia was significantly associated with uric acid level independent of indicators of renal function in the subjects. Abbreviation: PTH, parathyroid hormone. hyperparathyroidism) and Duh et $\mathrm{al}^{9}$ (143 patients operated on for primary hyperparathyroidism) found that the serum uric acid level significantly decreased in the patients who underwent parathyroidectomy. Christensson ${ }^{2}$ showed that the serum uric acid and calcium levels were significantly correlated in 41 patients with hyperparathyroidism, but the associations ceased to exist after parathyroidectomy. ${ }^{2}$ In the same study, ${ }^{2}$ no correlation between uric acid and PTH or calcium was found in age- and sex-matched normal subjects, which was a different result from the findings of the current study.

The association between parathyroid and uric acid is further evidenced by the use of teriparatide, a recombinant PTH used as antiosteoporotic agent. In the Fracture Prevention Trial, 1,638 postmenopausal women aged 42-86 years were given placebo or teriparatide $20 \mu \mathrm{g} /$ day or $40 \mu \mathrm{g}$ /day with calcium and vitamin D. ${ }^{5}$ The study showed that teriparatide increased the incidence of hyperuricemia in a dosedependent manner. ${ }^{5}$ Furthermore, the incidence was inflated in patients with moderate renal impairment. ${ }^{5}$ However, the incidence of gout was not increased. ${ }^{5}$ Hyperuricemia with or without gout was also reported as a side-effect in other teriparatide trials. ${ }^{10,11}$

The specific mechanism underlying the association between uric acid and PTH levels is currently unclear. The transport of uric acid involves secretory and absorptive transporters at the basolateral and apical membranes. ${ }^{12} \mathrm{PTH}$ is known to affect certain transporters in the proximal tubule of the kidney; for example, its inhibition of sodium/hydrogen ion exchanger type 3 isoform is known. ${ }^{13}$ We suggest that PTH is able to affect the transport of uric acid in the proximal tubule in the kidney through a presently unknown mechanism. In addition, it has been proposed that the renal secretion of uric acid is decreased in hyperuricemic subjects with hyperparathyroidism and that the severity depends on the alteration in reabsorption. ${ }^{14}$

There are several limitations to the current study. A non-randomized sampling technique was adopted, and only Chinese and Malay men were recruited for this study; hence, the results might not be representative of the whole Malaysian population. The use of allopurinol and uricosuric agents, was self-reported by the patients, and the occurrence of gout was assessed by a qualified physician during the screening session. The glomerular filtration rate of the subjects was not assessed in this study. The inaccuracy of these data might alter the strength of the relationship between uric acid and PTH, presumably toward a weaker one. Due to the cross-sectional nature of this study, the causal relationship between PTH and uric acid could not be established. It is possible that the observed relationship could 
be explained by co-association between uric acid and PTH with an unmeasured confounding variable. Despite these limitations, the current research was the first study to assess the relationship between circulating uric acid and PTH in the Asian population. Increased PTH level might predispose an individual to hyperuricemia, and the clinical importance of this finding should be further investigated.

\section{Conclusion}

Increased PTH is associated with increased uric acid and increased probability of being hyperuricemic. The relationship should be further evaluated in a Mendelian randomization study in addition to a longitudinal study.

\section{Acknowledgment}

We thank Universiti Kebangsaan Malaysia for funding this study via grants UKM-AP-TKP-09-2009, DIP-2014-40, and GGPM-2015-036.

\section{Disclosure}

The authors report no conflicts of interest in this work.

\section{References}

1. Smith E, Hoy D, Cross M, et al. The global burden of gout: estimates from the Global Burden of Disease 2010 study. Ann Rheum Dis. 2014;73(8): 1470-1476.

2. Christensson T. Serum urate in subjects with hypercalcaemic hyperparathyroidism. Clin Chim Acta. 1977;80(3):529-533.
3. Mintz DH, Canary JJ, Carreon G, Kyle LH. Hyperuricemia in hyperparathyroidism. N Engl J Med. 1961;265:112-115.

4. Bergenfelz A, Bladström A, Their M, Nordenström E, Valdemarsson S, Westerdahl J. Serum levels of uric acid and diabetes mellitus influence survival after surgery for primary hyperparathyroidism: a prospective cohort study. World J Surg. 2007;31(7):1393-1400; discussion 1401-1402.

5. Miller PD, Schwartz EN, Chen P, Misurski DA, Krege JH. Teriparatide in postmenopausal women with osteoporosis and mild or moderate renal impairment. Osteoporos Int. 2007;18(1):59-68.

6. Hui JY, Choi JW, Mount DB, Zhu Y, Zhang Y, Choi HK. The independent association between parathyroid hormone levels and hyperuricemia: a national population study. Arthritis Res Ther. 2012;14(2):R56.

7. Paik JM, Farwell WR, Taylor EN. Demographic, dietary, and serum factors and parathyroid hormone in the National Health and Nutrition Examination Survey. Osteoporos Int. 2012;23(6):1727-1736.

8. Chin KY, Ima-Nirwana S, Ibrahim S, Mohamed IN, Wan Ngah WZ. Vitamin D status in Malaysian men and its associated factors. Nutrients. 2014;6(12):5419-5433.

9. Duh QY, Morris RC, Arnaud CD, Clark OH. Decrease in serum uric acid level following parathyroidectomy in patients with primary hyperparathyroidism. World J Surg. 1986;10(4):729-736.

10. Saag KG, Shane E, Boonen S, et al. Teriparatide or alendronate in glucocorticoid-induced osteoporosis. N Engl J Med. 2007;357(20): 2028-2039.

11. Tsujimoto M, Uenaka K, Iwata A, Higashiuchi Y, Sowa H. Effects of teriparatide in Japanese and non-Japanese populations: bridging findings on pharmacokinetics and efficacy. J Bone Miner Metab. 2012;30(3): 326-337.

12. Lipkowitz MS. Regulation of uric acid excretion by the kidney. Curr Rheumatol Rep. 2012;14(2):179-188.

13. Girardi AC, Titan SM, Malnic G, Reboucas NA. Chronic effect of parathyroid hormone on NHE3 expression in rat renal proximal tubules. Kidney Int. 2000;58(4):1623-1631.

14. Hisatome I, Ishimura M, Sasaki N, et al. Renal handling of urate in two patients with hyperuricemia and primary hyperparathyroidism. Intern Med. 1992;31(6):807-811.
Clinical Interventions in Aging

\section{Publish your work in this journal}

Clinical Interventions in Aging is an international, peer-reviewed journal focusing on evidence-based reports on the value or lack thereof of treatments intended to prevent or delay the onset of maladaptive correlates of aging in human beings. This journal is indexed on PubMed Central, MedLine,

\section{Dovepress}

CAS, Scopus and the Elsevier Bibliographic databases. The manuscript management system is completely online and includes a very quick and fair peer-review system, which is all easy to use. Visit http://www.dovepress. com/testimonials.php to read real quotes from published authors. 